\title{
Discussing Fuzzy Reliability Estimators of Function of Mixed Probability Distribution by Simulation
}

\author{
Mohammed Kadhim Hawash
}

Received 8/11/2018, Accepted12/6/2019, Published 1/3/2020

This work is licensed under a Creative Commons Attribution 4.0 International License.

\begin{abstract}
:
This paper deals with constructing mixed probability distribution from exponential with scale parameter $(\beta)$ and also Gamma distribution with $(2, \beta)$, and the mixed proportions are $\left(\frac{\alpha}{\alpha+1}, \frac{1}{\alpha+1}\right)$.first of all, the probability density function (p.d.f) and also cumulative distribution function (c.d.f) and also the reliability function are obtained. The parameters of mixed distribution, $(\alpha, \beta)$ are estimated by three different methods, which are maximum likelihood, and Moments method,as well proposed method (Differential Least Square Method)(DLSM).The comparison is done using simulation procedure, and all the results are explained in tables.
\end{abstract}

Key words: Maximum Likelihood Estimator(MLE), Mean square error(MSE), Mixed Probability Distribution (MPD), Moment estimator(MOM), Proposed Method (DLSM).

\section{Introduction:}

The compound and mixture distributions provide a mathematical approach for doing statistical modeling different verity random phenomena because the mixture distribution are flexible models for and lyzing random durations, in the heterogeneous population. Also, the mixture distributions have vital role in practical applications for research that deal with economics, medicine, agriculture, life testing, and reliability for classical reliability theory. There are several methods and models in which the parameters assume precise, but in real world application, due to vague and randomness affect the life times distribution, also when the parameters of life time distribution are fuzzy, then there is difficulty for handling reliability and hazard functions Many researches work on fuzzy reliability and introduce development for this field, as this in dictated. In (2017), (1)two researchers (M.A. Hussian) and (Esssam A A. min) discussed fuzzy exponential distribution and discussed how to compute reliability in case of stress- strength model and ranked set sampling.Also in (2013) (2), two researchers (Elbatal) and (M.elgarhy) introduced Transmuted Quasi Lindley Distribution and worked on deriving $\left(r_{t h}\right)$ moment and moment generating function.

Discussing Fuzzy Reliability Estimators of Function of Mixed Probability Distribution by Simulation, Dijlah University College, Baghdad, Iraq.

E-mail:Muhamed.khadim@duc.edu.iq

*ORCID ID: 0000-0002-9836-130
In (2010) (3), (Mohamoudi and Zakerzaadeh) worked on generalized PoissonLindley Distribution and introduced different methods of estimation and comparing results by MSE, while in 2016 (4) (Nedjar and Zeghdoudi) worked on deriving gamma Lindley Distribution and studied its properties by Simulation. In (1970) (5) (Sankaran, M.) studied discrete Poisson- Lindley Distribution, and discussed its estimat. Also in (2013) (6) (Shanker and Mishra) introduced a research about quasi lindley distribution to Journal of Mathematics and Computer Science.

A Generalization of Lindley Distribution was introduced by (Zakerzadeh and Dolati) (2009) (7). Also (Zeghdoudi and Nedjar) in 2016 (8) introduced properties and application on Poisson Gamma Lindley Distribution. In May -Jun. (2015) (9) (Dutta and Borah) introduced a study about Poisson -Quasi Lindley Distribution, and derived its mathematical properties of coefficient of skewness and kurtosis coefficient of variation. In (2017) (10), (Rama Shanker and etl.) discuss three parameters Lindley,and studied their different estimators of parameter and reliability function, all mathematical and statistical properties were discussed. The aim of this research is to build mixed failure to time model from exponential and Gamma distribution where this model is necessary when the observation of time to failure cannot be represented by single probability distribution, which is recognized by scatter diagram, so two types of 
distributions need to be mixed which are exponential and Gamma using certain proportion as weights function, and the sum of this weight equals one.

\section{Theoretical Parts:}

Let $x$ be random variable distribution exponential with scale $\operatorname{parameter}(\beta)$ is defined in equation(1) as:

$$
h_{1}(x)=\beta e^{-\beta x} x>0, \beta>0
$$

Also there is another random variable $(y=x)$ distributionas Gamma $(2, \beta)$ as exponential in equation (2):

$$
h_{2}(x)=\beta^{2} x e^{-\beta x} x>0, \beta>0
$$

Then Mixing p.d.f of (1) and (2) by applying proportion $\left(\frac{\alpha}{\alpha+1}, \frac{1}{\alpha+1}\right)$ leads to:

$$
\begin{gathered}
f(x, \alpha, \beta)=\frac{\alpha}{\alpha+1} h_{1}(x)+\frac{1}{\alpha+1} h_{2}(x) \\
f(x, \alpha, \beta)=\frac{\alpha \beta}{\alpha+1} e^{-\beta x}+\frac{1}{\alpha+1} \beta^{2} x e^{-\beta x} \\
f(x, \alpha, \beta)=\frac{\beta}{\alpha+1} e^{-\beta x}(\alpha+\beta x) \quad \alpha \\
>-1 \ldots(4) \\
\beta>0 \\
x>0
\end{gathered}
$$

This p.d.f $f(x, \alpha, \beta)$ represent Quasi Lindy distribution with two parameters

( $\beta$ : scale parameter)

And

( $\alpha$ : is (mixed proportion) parameter)

The cumulative distribution function (C.D.F) correspoinal to p.d.f in equation (4) is:

$$
\begin{aligned}
F_{x}(x, \alpha, \beta)=\operatorname{pr} & (X \leq x) \\
& =\int_{0}^{x} f(t, \alpha, \beta) d t \\
& =1-\frac{(1+\alpha+\beta x) e^{-\beta x}}{(\alpha+1)}
\end{aligned}
$$

While Reliability Function is:

$$
R_{x}(x)=\frac{(1+\alpha+\beta x)}{(\alpha+1)} e^{-\beta x}
$$

and hazard rate function:

$$
\begin{array}{r}
h(x)=\frac{f(x)}{R(x)} \\
=\frac{\frac{\beta e^{-\beta x}(\alpha+\beta x)}{(\alpha+1)}}{\frac{(1+\alpha+\beta x)}{(\alpha+1)} e^{-\beta x}} \\
h(x)=\frac{\beta(\alpha+\beta x)}{(1+\alpha+\beta x)}
\end{array}
$$

and the fuzzy hazard Rate function

$\tilde{h}(x)$

$=\frac{\hat{\beta}\left(\hat{\alpha}+\hat{\beta} \hat{k}_{i} x_{i}\right)}{\left(1+\hat{\alpha}+\hat{\beta} \hat{k}_{i} x_{i}\right)} \ldots$...(8) , $\hat{k}_{i}$ is vague factor

\section{Estimation Method:}

In this section, some methods are presented for estimating $(\alpha, \beta)$ by different ways like moments, maximum likelihood, and differential least squares method

(D L S M) (proposed method).

The First Method: (Moment Method):

First of all, the formula of the moments about origin is derived from

$$
\mu r^{\prime}=E\left(x^{r}\right)=\int_{0}^{\infty} x^{r} f(x, \alpha, \beta) d x
$$

After some steps we find

$\mu r^{\prime}=\frac{\Gamma(r+1)(\alpha+r+1)}{\beta^{r}(\alpha+1)}$

Then from

$\dot{\mu}_{1=} \frac{\sum x i}{n}=\frac{\Gamma(2)(\alpha+2)}{\beta(\alpha+1)}=\bar{x}$

And from $\dot{\mu}_{1=} \frac{\sum x i^{2}}{n}$

$E\left(x^{2}\right)=\frac{2(\alpha+3)}{\beta^{2}(\alpha+1)}$

Then, from equation of variance which is

$$
\sigma^{2}=E\left(x^{2}\right)-(E x)^{2}
$$$$
=\frac{\alpha^{2}+4 \alpha+2}{\beta^{2}(\alpha+1)^{2}}
$$

the Coefficient of Variable (C.V) is:

$$
\begin{aligned}
(C . V) & =\frac{\sigma}{\mu_{1}} \\
= & \frac{\sqrt{\alpha^{2}+4 a+2}}{2 \alpha^{2}+2}
\end{aligned}
$$

where the $(C . V)$ is function of $\alpha$, so can equate (C.V) from sample with equation (10), and solving resulted equation, $\widehat{\alpha}_{\text {mom }}$ is obtained then gives $\hat{\beta}_{\text {mom }}$

$\hat{\beta}_{\text {mom }}=\sqrt{\frac{2 n(\widehat{\alpha}+3)}{\sum_{i=1}^{n} x i^{2}(\widehat{\alpha}+1)}}$

The Second Method: Maximum Likelihood Estimator(MLE):

The estimation on by this method depends on maximizing

$$
\begin{aligned}
L & =\prod_{i=1}^{n} f(x i, \alpha, \beta) \\
& =\beta^{n}(\alpha+1)^{-n} e^{-\beta \sum_{i=1}^{n} x i} \prod_{i=1}^{n}(\alpha+\beta x i)
\end{aligned}
$$

$\log L=\mathrm{n} \quad \log \beta-n \log (\alpha+1)-\beta \sum_{i=1}^{n} x i+$ $\sum_{i=1}^{n} \log (\alpha+\beta x i)$

$\frac{\partial \log L}{\partial \beta}=\frac{n}{\beta}-\sum_{i=1}^{n} x i+\sum_{i=1}^{n} \frac{x i}{(\alpha+\beta x i)}$

$\frac{\partial \log L}{\partial \alpha}=\frac{-n}{(\alpha+1)}+\sum_{i=1}^{n} \frac{1}{(\alpha+\beta x i)}$

from $\frac{\partial \log L}{\partial \beta}=0 \rightarrow$ 
$\frac{n}{\hat{\beta}}=\sum_{i=1}^{n} x i-\sum_{i=1}^{n} \frac{x i}{(\hat{\alpha}+\hat{\beta} x i)}$

$\hat{\beta}_{M L E}=\frac{n}{\sum_{i=1}^{n} x i-\sum_{i=1}^{n} \frac{x i}{(\widehat{\alpha}+\widehat{\beta} x i)}}$

and from $\frac{\partial \log L}{\partial \alpha}=0$

$\sum_{i=1}^{n}(\hat{\alpha}+\widehat{\beta} x i)^{-1}=\frac{n}{\hat{\alpha}+1}$

$\widehat{\alpha}+1=\frac{n}{\sum_{i=1}^{n}(\hat{\alpha}+\hat{\beta} x i)^{-1}}$

from equation $\hat{\beta}_{M L E}, \hat{\beta}_{M L E}$ is found to be an implicit function from $(\hat{\alpha}, \hat{\beta})$ so iterative procedure can be used according to the generated values of $(\mathrm{Xi}$, using assumed $\alpha, \beta$ ) to find

$\left|\hat{\beta}_{i+1 M L E}-\hat{\beta}_{i M L E}\right|<$ to lerance also $\hat{\alpha}$ is found using same procedur.

$\hat{\alpha}_{M L E}=\frac{n}{\sum_{i=1}^{n}(\widehat{\alpha}+\widehat{\beta} x i)^{-1}}-1 \ldots$

The Third method: (Differential Least Square Method (DLSM):

It is assumed here that data sets, are generated from Quasi Lindlly with tow parameters ( $\alpha$ and $\beta$ ), since $\beta$ is a scale parameter it can be estimated by (DLSM), assuming that ( $\alpha$ is known). Also the two parameters ( $\alpha$ and $\beta$ ) are estimated Moments, and Maximum likelihood, as explained previously.

$$
\begin{aligned}
& f(x)=\left(\frac{\beta}{\alpha+1}\right) e^{-\beta x}(\alpha+\beta x) \\
& =\left(\frac{1}{\alpha+1}\right) e^{-\beta x}\left(\alpha \beta+\beta^{2} x\right) \\
& \frac{d f}{d x}=\left(\frac{1}{\alpha+1}\right)\left[e^{-\beta x}\left(\beta^{2}\right)\right. \\
& \left.+\left(\alpha \beta+\beta^{2} x\right) e^{-\beta x}(-\beta)\right] \\
& =\left(\frac{1}{\alpha+1}\right) \beta^{2}\left[e^{-\beta x}-(\alpha+\beta x) e^{-\beta x}\right] \\
& \frac{d^{2} f}{d x^{2}}=\frac{\beta^{2}}{(\alpha+1)}\left[-\beta e^{-\beta x}-(\alpha+\beta x) e^{-\beta x}(-\beta)\right. \\
& \left.+e^{-\beta x}(\beta)\right] \\
& =\frac{\beta^{2}}{(\alpha+1)}\left[-\boldsymbol{\beta} e^{-\beta x}+\beta e^{-\beta x}(\alpha+\beta x)\right. \\
& \left.-\beta e^{-\beta x}\right] \\
& =\frac{\beta^{2}}{(\alpha+1)}\left[\beta e^{-\beta x}(\alpha+\beta x)-2 \beta e^{-\beta x}\right] \\
& \therefore f(x)=\left(\frac{\beta}{\alpha+1}\right) e^{-\beta x}(\alpha+\beta x)
\end{aligned}
$$

There for

$\frac{d^{2} f}{d x^{2}}=\left[\beta^{2} f(x)-2 \frac{\beta^{3}}{\alpha+1} e^{-\beta x}\right]$

The scale parameter $\beta$ can be obtained by Minimizing

$$
\begin{array}{r}
T=\sum_{i=1}^{n}\left[\beta^{2} f(x i)-\frac{2 \beta^{3}}{\alpha+1} e^{-\beta x i}\right. \\
-\hat{f}(x i)]^{2} \ldots(13)
\end{array}
$$

Using instruction (f: solve)in math lab

\section{Simulation Procedures:}

The following three steps are concluded:

1-Generate values of random $\sim$ (Quasi Lindely) with (Parameters $(\alpha+\beta))$ using rejection and accept method.

2- Generate random variable $\mathrm{Zi} \sim$ uniform $(0,1)$

3- Generate two random variable $V i \sim \exp \beta$ ), Wi $\sim \operatorname{Gamma}(2, \beta)$

If $\quad u i \leq p=\frac{\alpha i}{\alpha+1}$ then $x i=v i$

other wise $\quad x i=w i, \quad x>0$

$$
\begin{gathered}
\beta>0 \\
\alpha>-1
\end{gathered}
$$

And by inverse transformation from

$F_{x}(x i, \alpha, \beta)=1-\frac{(1+\alpha+\beta x i) e^{-\beta x i}}{(\alpha+1)}$

$u i=1-\frac{(1+\alpha+\beta x i) e^{-\beta x i}}{(\alpha+1)}$

$\frac{(1+\alpha+\beta x i)}{(\alpha+1)}=(1-u i)$

$1+\frac{\beta}{(\alpha+1)} x i=(1-u i)$

$u i=-\frac{\beta}{(\alpha+1)} x i$

$\log u i=\left(-\frac{\beta}{\alpha+1}\right) x i, \theta=-\frac{\beta}{\alpha+1} \alpha>-1$

$\log u i=\theta x i \quad, 0<x i<\infty$

$x i=\frac{u i}{\theta}$

According to the given values of $(\beta, \alpha)$ and $(0 \leq u i \leq 1)$, values of $(X i)$ are generated These observation are generated according to different sample size $n=(25,50,80)$.

There are different values for $(\alpha=0.8,1.2)$, $(\beta=1.5,2.5)$ and $(\tilde{k}=0.3,0.7)$

And each experiment is repeated $R=500$

All the results of comparisons of fuzzy reliability functions, are explained in different Tables( $1,2,3,4,5,6,7,8)$ and according to different sets of initial values. The comparison is done using statistical Measures Mean Square Error.

The values of variable $(x i)$, are generate by simulation, using different sets of sample size $(n=$ $25,50,80)$ and also different sets of initial values of parameters which are $(\alpha=0.8,1.2), \quad(\beta=$ $1.5,2.5)$ and vague factor $(\tilde{k}=0.3,0.7)$. 
Table 1. Estimator Fuzzy Reliability when $\beta=1.5, \alpha=0.8, \quad \tilde{k}=0.3$

\begin{tabular}{|c|c|c|c|c|c|c|c|c|c|}
\hline $\mathbf{n}$ & $\boldsymbol{t}_{i}$ & Rreal & $\boldsymbol{R}_{\text {mle }}$ & $\boldsymbol{R}_{\text {mom }}$ & $R_{\text {pro }}$ & mse-mle & mse-mom & mse-pro & Best \\
\hline & 0.0272 & 0.946049 & 0.97691 & 0.785567 & 0.999292 & 0.059432 & 0.736766 & 0.056627 & mse-pro \\
\hline & 0.1176 & 0.825691 & 0.935831 & 0.780076 & 0.984853 & 0.053082 & 0.050132 & 0.383169 & mse-mom \\
\hline & 0.2636 & 0.801620 & 0.909449 & 0.780059 & 0.920505 & $3.15 \mathrm{E}-02$ & 0.323819 & 0.035277 & mse-mle \\
\hline & 0.2763 & 0.801321 & 0.844168 & 0.777832 & 0.912604 & 0.025516 & 0.320932 & 0.033574 & mse-mle \\
\hline \multirow[t]{10}{*}{25} & 0.3065 & 0.786306 & 0.839904 & 0.764597 & 0.892580 & $7.08 \mathrm{E}-03$ & 0.299041 & 0.023326 & mse-mle \\
\hline & 0.3156 & 0.777789 & 0.835302 & 0.750762 & 0.886178 & 0.002859 & 0.279858 & 0.018034 & mse-mle \\
\hline & 0.4304 & 0.775390 & 0.828518 & 0.747624 & 0.793320 & 0.002068 & 0.259567 & $1.60 \mathrm{E}-02$ & mse-mle \\
\hline & 0.4870 & 0.758288 & 0.827583 & 0.747442 & 0.740868 & $2.06 \mathrm{E}-03$ & 0.239961 & 0.015138 & mse-mle \\
\hline & 0.5508 & 0.698358 & 0.799396 & 0.709178 & 0.678283 & $1.68 \mathrm{E}-03$ & 0.175245 & 0.013039 & mse-mle \\
\hline & 0.5654 & 0.682541 & 0.792465 & 0.589471 & 0.663601 & 0.157895 & 8.46E-04 & 7.19E-03 & mse-mom \\
\hline & 0.1130 & 0.957881 & 0.993002 & 0.745025 & 0.97914 & 0.014515 & 0.778453 & 0.026833 & mse-mle \\
\hline & 0.1170 & 0.925756 & 0.960244 & 0.721437 & 0.977799 & 0.01448 & 0.658603 & 0.022971 & mse-mle \\
\hline & 0.1180 & 0.897648 & 0.949353 & 0.720171 & 0.977548 & $1.45 \mathrm{E}-02$ & 0.529383 & 0.018778 & mse-mle \\
\hline & 0.1400 & 0.896738 & 0.937497 & 0.713703 & 0.969649 & 0.523588 & 0.010281 & 0.01031 & mse-mom \\
\hline \multirow[t]{12}{*}{50} & 0.1429 & 0.876332 & 0.932636 & 0.696337 & 0.968338 & $5.25 \mathrm{E}-03$ & 0.50743 & 0.010234 & mse-mle \\
\hline & 0.1554 & 0.847696 & 0.920616 & 0.686673 & 0.963216 & 0.004862 & 0.430279 & 0.006763 & mse-mle \\
\hline & 0.1660 & 0.843932 & 0.907975 & 0.682311 & 0.958572 & 0.00246 & 0.407352 & $6.64 \mathrm{E}-03$ & mse-mle \\
\hline & 0.1860 & 0.840514 & 0.905161 & 0.664759 & 0.949236 & $5.40 \mathrm{E}-04$ & 0.404829 & 0.005127 & mse-mle \\
\hline & 0.1944 & 0.826236 & 0.894905 & 0.614678 & 0.945096 & $1.82 \mathrm{E}-04$ & 0.379749 & 0.000138 & mse-pro \\
\hline & 0.2149 & 0.799407 & 0.877087 & 0.577119 & 0.934418 & $7.50 \mathrm{E}-05$ & 0.320517 & $5.55 \mathrm{E}-06$ & mse-mle \\
\hline & 0.0090 & 0.969321 & 0.982877 & 0.689771 & 0.999148 & 0.011761 & 0.831525 & 0.013222 & mse-mle \\
\hline & 0.0312 & 0.954974 & 0.977239 & 0.689001 & 0.994266 & 0.005293 & 0.742213 & 0.009864 & mse-pro \\
\hline & 0.0477 & 0.947730 & 0.975136 & 0.674799 & 0.988969 & 0.00925 & 0.72747 & $3.24 \mathrm{E}-03$ & mse-mle \\
\hline & 0.0494 & 0.944398 & 0.942233 & 0.671358 & 0.988384 & 0.001855 & 0.717598 & 0.009228 & mse-pro \\
\hline & 0.0539 & 0.931762 & 0.941943 & 0.646874 & 0.986719 & $1.79 \mathrm{E}-03$ & 0.005465 & 0.643143 & mse-mom \\
\hline & 0.0555 & 0.921143 & 0.930798 & 0.621636 & 0.986123 & 0.001686 & 0.641488 & 0.0043 & mse-pro \\
\hline \multirow[t]{4}{*}{80} & 0.1425 & 0.902972 & 0.928522 & 0.603719 & 0.941921 & 0.000498 & 0.541825 & 7.89E-04 & mse-mle \\
\hline & 0.1682 & 0.894948 & 0.911721 & 0.600688 & 0.925659 & $2.28 \mathrm{E}-04$ & 0.000487 & 0.537192 & mse-mom \\
\hline & 0.2005 & 0.892319 & 0.911695 & 0.597813 & 0.903666 & .000386 & 0.520319 & $6.47 \mathrm{E}-050$ & mse-mle \\
\hline & 0.2318 & 0.877676 & 0.851887 & 0.558438 & 0.88105 & $6.13 \mathrm{E}-06$ & 0.491288 & $4.78 \mathrm{E}-05$ & mse-mle \\
\hline
\end{tabular}

Table 2. Estimator Fuzzy Reliability when $\beta=1.5, \alpha=1.2, \tilde{k}=0.3$

\begin{tabular}{|c|c|c|c|c|c|c|c|c|c|}
\hline $\mathbf{n}$ & $t_{i}$ & Rreal & $R_{m l e}$ & $\boldsymbol{R}_{\text {mom }}$ & $R_{\text {pro }}$ & mse-mle & mse-mom & mse-pro & Best \\
\hline \multirow{8}{*}{25} & 0.0356 & 0.946049 & 0.957776 & 0.835522 & 0.999168 & 0.050506 & 0.684143 & 0.037605 & mse-pro \\
\hline & 0.1540 & 0.825691 & 0.890355 & 0.830157 & 0.982229 & 0.035319 & 0.329527 & 0.050077 & mse-mle \\
\hline & 0.3450 & 0.80162 & 0.868772 & 0.826366 & 0.907276 & $2.86 \mathrm{E}-02$ & 0.275702 & 0.033925 & mse-mle \\
\hline & 0.3620 & 0.801321 & 0.829565 & 0.824991 & 0.898134 & 0.032620 & 0.27198 & 0.018972 & mse-pro \\
\hline & 0.4016 & 0.786306 & 0.828043 & 0.810744 & 0.875028 & 0.250062 & $4.14 \mathrm{E}-03$ & 0.028663 & mse-mom \\
\hline & 0.4136 & 0.777789 & 0.82297 & 0.797465 & 0.86766 & 0.001703 & 0.232625 & 0.028066 & mse-mle \\
\hline & 0.5640 & 0.77539 & 0.818081 & 0.792971 & 0.761861 & $2.56 \mathrm{E}-02$ & 0.214717 & 0.001535 & mse-pro \\
\hline & 0.6382 & 0.758288 & 0.816586 & 0.791864 & 0.703033 & $9.11 \mathrm{E}-04$ & 0.196189 & 5045 & mse-mle \\
\hline \multirow{13}{*}{50} & 7217 & 0.698358 & 0.799875 & 0.754682 & 0.633793 & 0.135772 & $8.80 \mathrm{E}-04$ & 3628 & mse-mom \\
\hline & 409 & 0.682541 & 0.799746 & 0.621953 & 0.617708 & 8.0 & & & \\
\hline & 1490 & 0.957881 & 0.98909 & 0.791153 & 0.977218 & 0.013709 & 0.7 & 744 & mse-mle \\
\hline & 540 & 0.925756 & 0.949153 & 0.765381 & 0.975755 & & & & mse-mom \\
\hline & 0.1550 & 0.897648 & 0.944543 & 0.762237 & 0.975482 & $1.16 \mathrm{E}-02$ & 0.4 & 216 & mse-mle \\
\hline & 0.1830 & 0.896738 & 0.926988 & 0.756952 & 0.966867 & 0.008248 & 76 & 0.009398 & mse-pro \\
\hline & 0.1873 & 0.876332 & 0.923504 & 0.740323 & 0.965439 & $4.66 \mathrm{E}-03$ & 0.446839 & 0.009255 & mse-pro \\
\hline & 0.2036 & 0.847696 & 0.917901 & 0.723021 & 0.959857 & 0.004264 & 0.375446 & 0.00 & mse-pro \\
\hline & 0.2175 & 0.843932 & 0.904422 & 0.721824 & 0.9548 & 0.357113 & & -03 & mse-mom \\
\hline & 0.2437 & 0.840514 & 0.902403 & 0.709172 & 0.944638 & $3.64 \mathrm{E}-04$ & 0.3 & 720 & mse-mle \\
\hline & 2547 & 0.826236 & 0.894512 & 0.650829 & 0.940135 & & & & mse-pro \\
\hline & 816 & 0.799407 & 0.881307 & 0.605311 & 0.928529 & 7.94E-06 & 0.270491 & 7.69E-06 & mse-pro \\
\hline & & & 0.978848 & 0.72 & 0.99 & & & & \\
\hline \multirow{9}{*}{80} & 409 & 74 & 0.973983 & 0.72 & 0.994202 & 0.6 & & 551 & mse-mom \\
\hline & 0.0626 & 0.94773 & 0.97067 & 0.713714 & 0.988846 & 2.69 & 0.6 & 248 & mse-mle \\
\hline & 0.0647 & 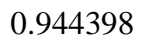 & 0.941513 & 0.710 & 0.988254 & & & & \\
\hline & 0.0706 & 0.931762 & 0.939375 & 0.678789 & 0.986571 & $1.75 \mathrm{E}-03$ & 0.597277 & 0.005652 & mse-mle \\
\hline & 0.0727 & 0.921143 & 0.931259 & 0.658526 & 0.985968 & 0.584735 & & 0.004281 & mse-mom \\
\hline & 0.1867 & 02972 & 0.928057 & 0.634207 & 0.941288 & 0.000416 & 0.4 & 8.48E-04 & mse-mle \\
\hline & 0.2204 & 0.894948 & 0.916283 & 0.631981 & 0.924855 & $3.22 \mathrm{E}-04$ & 0.484445 & 0.000523 & mse-pro \\
\hline & 2628 & 892319 & 0.91542 & 0.628 & 0.902638 & 0.4724 & 6.02E-05 & 3.81E-04 & mse-mom \\
\hline & 0.3037 & 0.877676 & 0.872582 & 0.582181 & 0.879797 & $2.44 \mathrm{E}-05$ & 0.439766 & $9.67 \mathrm{E}-06$ & mse-pro \\
\hline
\end{tabular}


Table 3. Estimator Fuzzy Reliability when $\beta=2.5, \alpha=0.8, \tilde{k}=0.3$

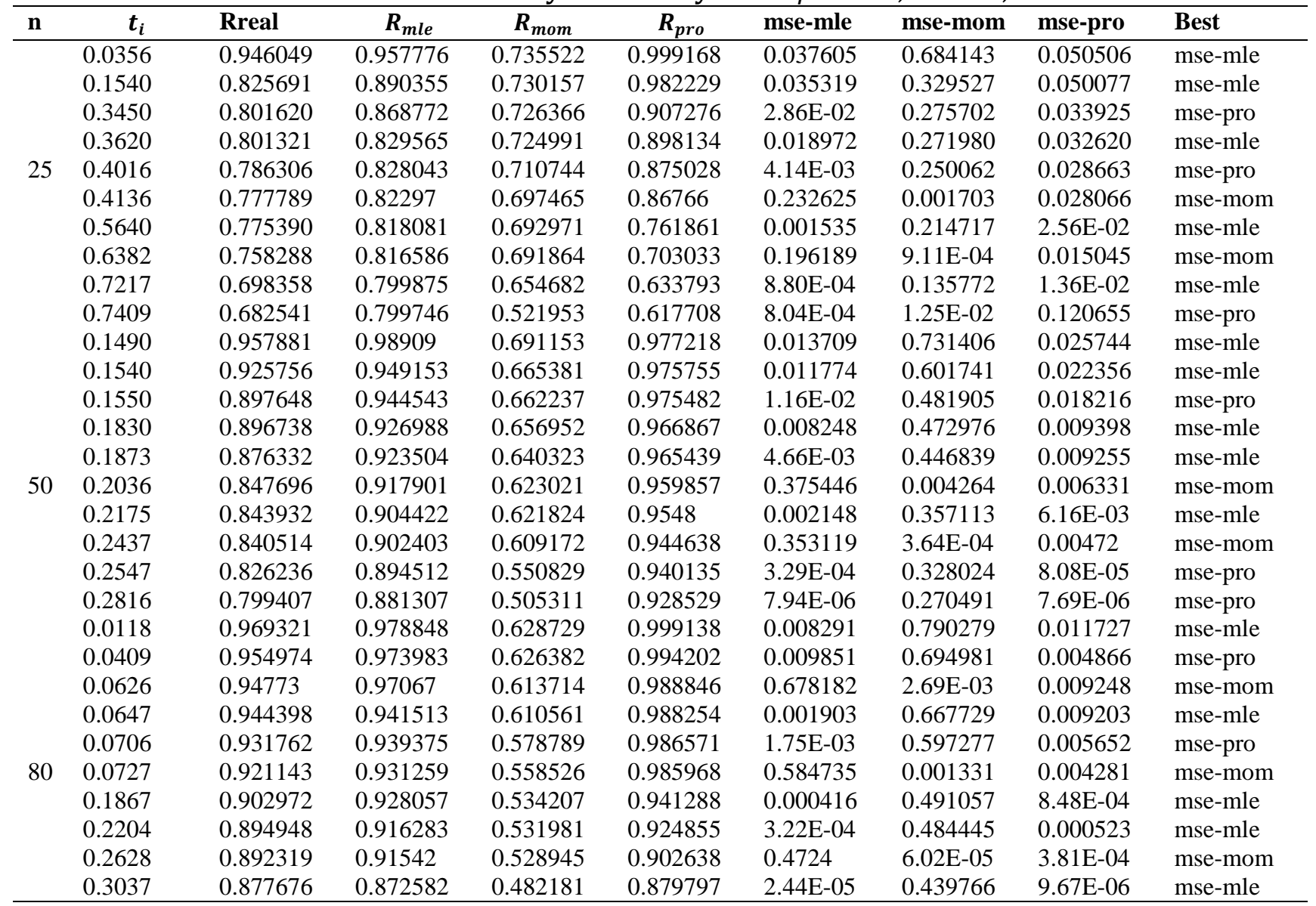

Table 4. Estimator Fuzzy Reliability when $\beta=2.5, \alpha=1.2, \tilde{k}=0.3$

\begin{tabular}{|c|c|c|c|c|c|c|c|c|c|}
\hline n & $t_{i}$ & Rreal & $R_{m l e}$ & $R_{\text {mom }}$ & $R_{\text {pro }}$ & mse-mle & mse-mom & mse-pro & Best \\
\hline \multirow{10}{*}{25} & 0.1350 & 0.946049 & 0.957776 & 0.806106 & 0.999071 & 0.037605 & 0.850763 & 0.050033 & mse-mle \\
\hline & 0.3260 & 0.825691 & 0.890355 & 0.792447 & 0.981117 & 0.035319 & 0.435708 & 0.049150 & mse-mle \\
\hline & 0.5280 & 0.801620 & 0.868772 & 0.736442 & 0.904239 & $2.86 \mathrm{E}-02$ & 0.372494 & 0.035261 & mse-pro \\
\hline & 0.5440 & 0.801321 & 0.829565 & 0.721052 & 0.894975 & 0.018972 & 0.371400 & 0.032219 & mse-mle \\
\hline & 0.5785 & 0.786306 & 0.828043 & 0.720283 & 0.871627 & 0.336924 & $4.14 \mathrm{E}-03$ & 0.028932 & mse-mom \\
\hline & 0.5887 & 0.777789 & 0.822970 & 0.709760 & 0.86420 & 0.001703 & 0.318107 & 0.027504 & mse-mle \\
\hline & 0.7092 & 0.775390 & 0.818081 & 0.695313 & 0.75827 & 0.312801 & 0.001535 & $2.64 \mathrm{E}-02$ & mse-mom \\
\hline & 0.7638 & 0.758288 & 0.816586 & 0.694722 & 0.699792 & $9.11 \mathrm{E}-04$ & 0.275060 & 0.015851 & mse-mle \\
\hline & 0.8223 & 0.698358 & 0.799874 & 0.665831 & 0.631228 & 8.80E-04 & $1.28 \mathrm{E}-02$ & 0.166598 & mse-pro \\
\hline & 0.8353 & 0.682541 & 0.799746 & 0.524129 & 0.615333 & 8.04E-04 & 0.143179 & $1.18 \mathrm{E}-02$ & mse-mle \\
\hline \multirow{10}{*}{50} & 0.3190 & 0.957881 & 0.98909 & 0.696275 & 0.975735 & 0.013709 & 0.890331 & 0.025061 & mse-mle \\
\hline & 0.3250 & 0.925756 & 0.949153 & 0.668325 & 0.974208 & 0.774819 & 0.011774 & 0.021896 & mse-mom \\
\hline & 0.3260 & 0.897648 & 0.944543 & 0.651369 & 0.973923 & $1.16 \mathrm{E}-02$ & 0.661552 & 0.017798 & mse-mle \\
\hline & 0.3610 & 0.896738 & 0.926988 & 0.649965 & 0.964966 & 0.008248 & 0.648514 & 0.008903 & mse-mle \\
\hline & 0.3660 & 0.876332 & 0.923504 & 0.641219 & 0.963486 & 0.008743 & 0.598803 & $4.66 \mathrm{E}-03$ & mse-pro \\
\hline & 0.3848 & 0.847696 & 0.917901 & 0.603245 & 0.957713 & 0.004264 & 0.500005 & 0.006098 & mse-mle \\
\hline & 0.4004 & 0.843932 & 0.904422 & 0.593858 & 0.952497 & 0.485212 & 0.002148 & $5.80 \mathrm{E}-03$ & mse-mom \\
\hline & 0.4287 & 0.840514 & 0.902403 & 0.584925 & 0.942052 & $3.64 \mathrm{E}-04$ & 0.476957 & 0.004455 & mse-mle \\
\hline & 0.4402 & 0.826236 & 0.894512 & 0.54555 & 0.937436 & $3.29 \mathrm{E}-04$ & 0.436495 & $5.02 \mathrm{E}-05$ & mse-pro \\
\hline & 0.4675 & 0.799407 & 0.881307 & 0.514413 & 0.925572 & 7.94E-06 & 0.368791 & $3.38 \mathrm{E}-08$ & mse-pro \\
\hline \multirow{10}{*}{80} & 0.0698 & 0.969321 & 0.978848 & 0.610637 & 0.999004 & 0.927656 & 0.008291 & 0.011459 & mse-mom \\
\hline & 0.1470 & 0.954974 & 0.973983 & 0.599582 & 0.993565 & 0.004866 & 0.867785 & 0.009725 & mse-mle \\
\hline & 0.1900 & 0.94773 & 0.97067 & 0.588242 & 0.987795 & 0.009222 & 0.851801 & $2.69 \mathrm{E}-03$ & mse-pro \\
\hline & 0.1930 & 0.944398 & 0.941513 & 0.578586 & 0.987161 & 0.001903 & 0.839705 & 0.008995 & mse-mle \\
\hline & 0.2039 & 0.931762 & 0.939375 & 0.555417 & 0.985364 & 0.772646 & $1.75 \mathrm{E}-03$ & 0.006338 & mse-mom \\
\hline & 0.2074 & 0.921143 & 0.931259 & 0.551491 & 0.984723 & 0.001331 & 0.756505 & 0.004124 & mse-mle \\
\hline & 0.3654 & 0.902972 & 0.928057 & 0.528129 & 0.938092 & $1.10 \mathrm{E}-03$ & 0.68055 & 0.000416 & mse-pro \\
\hline & 0.4036 & 0.894948 & 0.916283 & 0.524952 & 0.921216 & $3.22 \mathrm{E}-04$ & 0.652755 & 0.000703 & mse-mle \\
\hline & 0.4485 & 0.892319 & 0.91542 & 0.52473 & 0.89854 & 0.633093 & $6.02 \mathrm{E}-05$ & $3.41 \mathrm{E}-04$ & mse-mom \\
\hline & 0.4892 & 0.877676 & 0.872582 & 0.506231 & 0.875361 & $2.44 \mathrm{E}-05$ & 0.590866 & $3.98 \mathrm{E}-05$ & mse-mle \\
\hline
\end{tabular}


Table 5. Estimator Fuzzy Reliability when $\beta=1.5, \alpha=0.8, \tilde{k}=0.7$

\begin{tabular}{|c|c|c|c|c|c|c|c|c|c|}
\hline $\mathrm{n}$ & $t_{i}$ & Rreal & $R_{\text {mle }}$ & $R_{\text {mom }}$ & $R_{\text {pro }}$ & mse-mle & mse-mom & mse-pro & Best \\
\hline \multirow{10}{*}{25} & 0.0272 & 0.946049 & 0.976910 & 0.785567 & 0.999292 & 0.059432 & 0.736766 & 0.056627 & mse-pro \\
\hline & 0.1180 & 0.825691 & 0.935831 & 0.780076 & 0.984853 & 0.053082 & 0.383169 & 0.050132 & mse-pro \\
\hline & 0.2640 & 0.80162 & 0.909449 & 0.780059 & 0.920505 & $3.15 \mathrm{E}-02$ & 0.323819 & 0.035277 & mse-mle \\
\hline & 0.2760 & 0.801321 & 0.844168 & 0.777832 & 0.912604 & 0.320932 & 0.025516 & 0.033574 & mse-mom \\
\hline & 0.3065 & 0.786306 & 0.839904 & 0.764597 & 0.89258 & $7.08 \mathrm{E}-03$ & 0.299041 & 0.023326 & mse-mle \\
\hline & 0.3156 & 0.777789 & 0.835302 & 0.750762 & 0.886178 & 0.279858 & 0.002859 & 0.018034 & mse-mom \\
\hline & 0.4304 & 0.77539 & 0.828518 & 0.747624 & 0.79332 & $1.60 \mathrm{E}-02$ & 0.259567 & 0.002068 & mse-pro \\
\hline & 0.4870 & 0.758288 & 0.827583 & 0.747442 & 0.740868 & $2.06 \mathrm{E}-03$ & 0.239961 & 0.015138 & mse-mle \\
\hline & 0.5508 & 0.698358 & 0.799396 & 0.709178 & 0.678283 & 0.175245 & $1.68 \mathrm{E}-03$ & $1.30 \mathrm{E}-02$ & mse-mom \\
\hline & 0.5654 & 0.682541 & 0.792465 & 0.589471 & 0.663601 & $8.46 \mathrm{E}-04$ & 0.157895 & 7.19E-03 & mse-mle \\
\hline \multirow{11}{*}{50} & 0.1130 & 0.957881 & 0.993002 & 0.745025 & 0.97914 & 0.026833 & 0.778453 & 0.014515 & mse-pro \\
\hline & 0.1170 & 0.925756 & 0.960244 & 0.721437 & 0.977799 & 0.01448 & 0.658603 & 0.022971 & mse-mle \\
\hline & 0.1180 & 0.897648 & 0.949353 & 0.720171 & 0.977548 & 0.529383 & $1.45 \mathrm{E}-02$ & 0.018778 & mse-mom \\
\hline & 0.1400 & 0.896738 & 0.937497 & 0.713703 & 0.969649 & 0.01031 & 0.523588 & 0.010281 & mse-pro \\
\hline & 0.1429 & 0.876332 & 0.932636 & 0.696337 & 0.968338 & 0.50743 & $5.25 \mathrm{E}-03$ & 0.010234 & mse-mom \\
\hline & 0.1554 & 0.847696 & 0.920616 & 0.686673 & 0.963216 & 0.004862 & 0.430279 & 0.006763 & mse-mle \\
\hline & 0.1660 & 0.843932 & 0.907975 & 0.682311 & 0.958572 & 6.64E-03 & 0.407352 & 0.00246 & mse-pro \\
\hline & 0.1860 & 0.840514 & 0.905161 & 0.664759 & 0.949236 & $5.40 \mathrm{E}-04$ & 0.404829 & 0.005127 & mse-mle \\
\hline & 0.1944 & 0.826236 & 0.894905 & 0.614678 & 0.945096 & $1.82 \mathrm{E}-04$ & 0.379749 & $1.38 \mathrm{E}-04$ & mse-pro \\
\hline & 0.2149 & 0.799407 & 0.877087 & 0.677119 & 0.934418 & 5.55E-06 & 0.320517 & $7.50 \mathrm{E}-05$ & mse-mle \\
\hline & 0.0090 & 0.969321 & 0.982877 & 0.689771 & 0.999148 & 0.013222 & 0.831525 & 0.011761 & mse-pro \\
\hline \multirow{9}{*}{80} & 0.0312 & 0.954974 & 0.977239 & 0.689001 & 0.994266 & 0.005293 & 0.742213 & 0.009864 & mse-mle \\
\hline & 0.0477 & 0.94773 & 0.975136 & 0.674799 & 0.988969 & 0.72747 & $3.24 \mathrm{E}-03$ & 0.00925 & mse-mom \\
\hline & 0.0494 & 0.944398 & 0.942233 & 0.671358 & 0.988384 & 0.001855 & 0.717598 & 0.009228 & mse-mle \\
\hline & 0.0539 & 0.931762 & 0.941943 & 0.646874 & 0.986719 & 0.643143 & $1.79 \mathrm{E}-03$ & 0.005465 & mse-mom \\
\hline & 0.0555 & 0.921143 & 0.930798 & 0.621636 & 0.986123 & 0.004300 & 0.641488 & 0.001686 & mse-pro \\
\hline & 0.1425 & 0.902972 & 0.928522 & 0.603719 & 0.941921 & 0.000498 & 0.541825 & $7.89 \mathrm{E}-04$ & mse-mle \\
\hline & 0.1682 & 0.894948 & 0.911721 & 0.600688 & 0.925659 & 0.537192 & $2.28 \mathrm{E}-04$ & 0.000487 & mse-mom \\
\hline & 0.2005 & 0.892319 & 0.911695 & 0.597813 & 0.903666 & $6.47 \mathrm{E}-05$ & 0.520319 & $3.86 \mathrm{E}-04$ & mse-mle \\
\hline & 0.2318 & 0.877676 & 0.851887 & 0.558438 & 0.88105 & 4.78E-05 & 0.491288 & $6.13 \mathrm{E}-06$ & mse-pro \\
\hline
\end{tabular}

Table 6. Estimator Fuzzy Reliability when $\beta=1.5, \alpha=1.2, \tilde{k}=0.7$

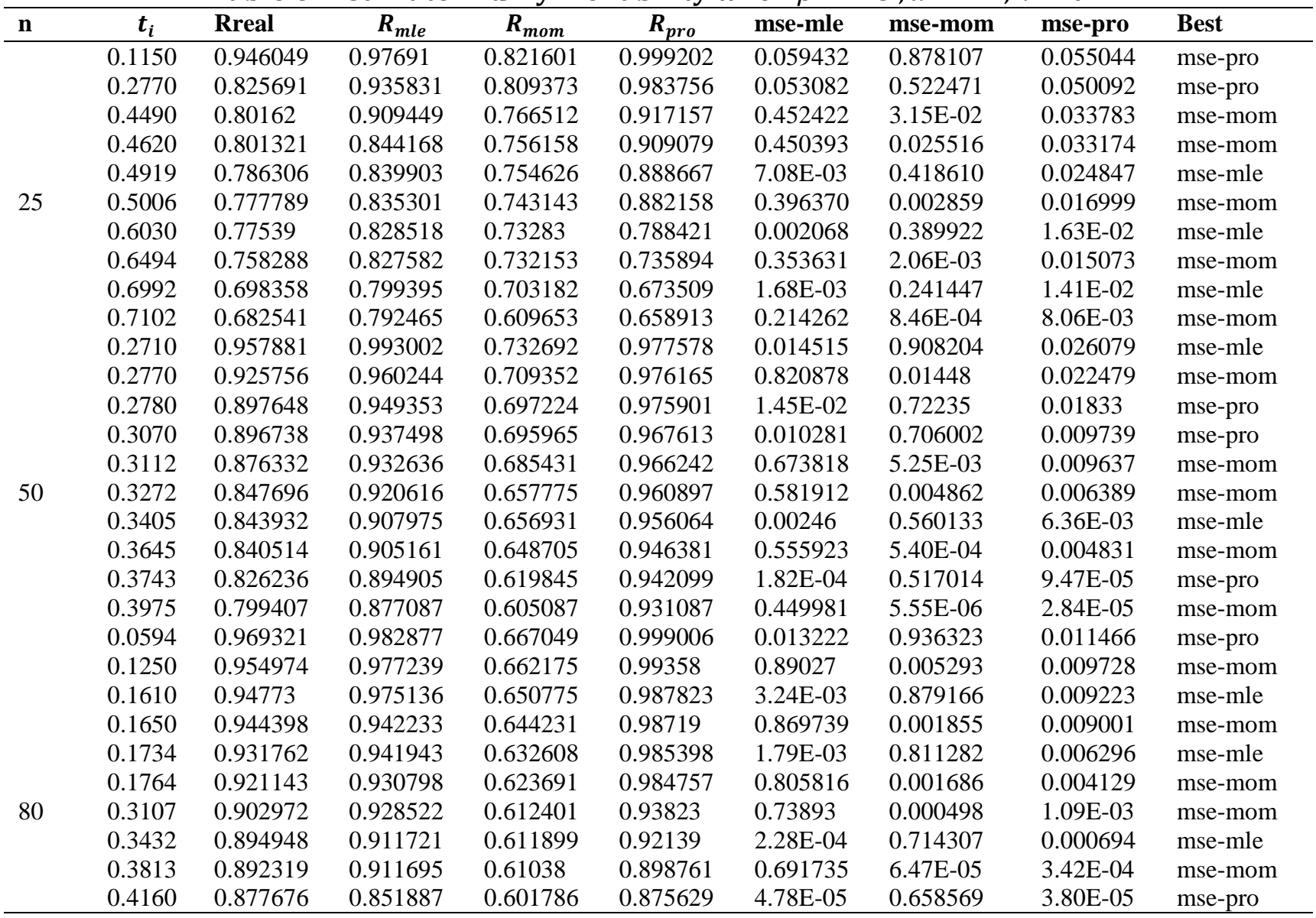


Table 7. Estimator Fuzzy Reliability when $\beta=2.5, \alpha=0.8, \tilde{k}=0.7$

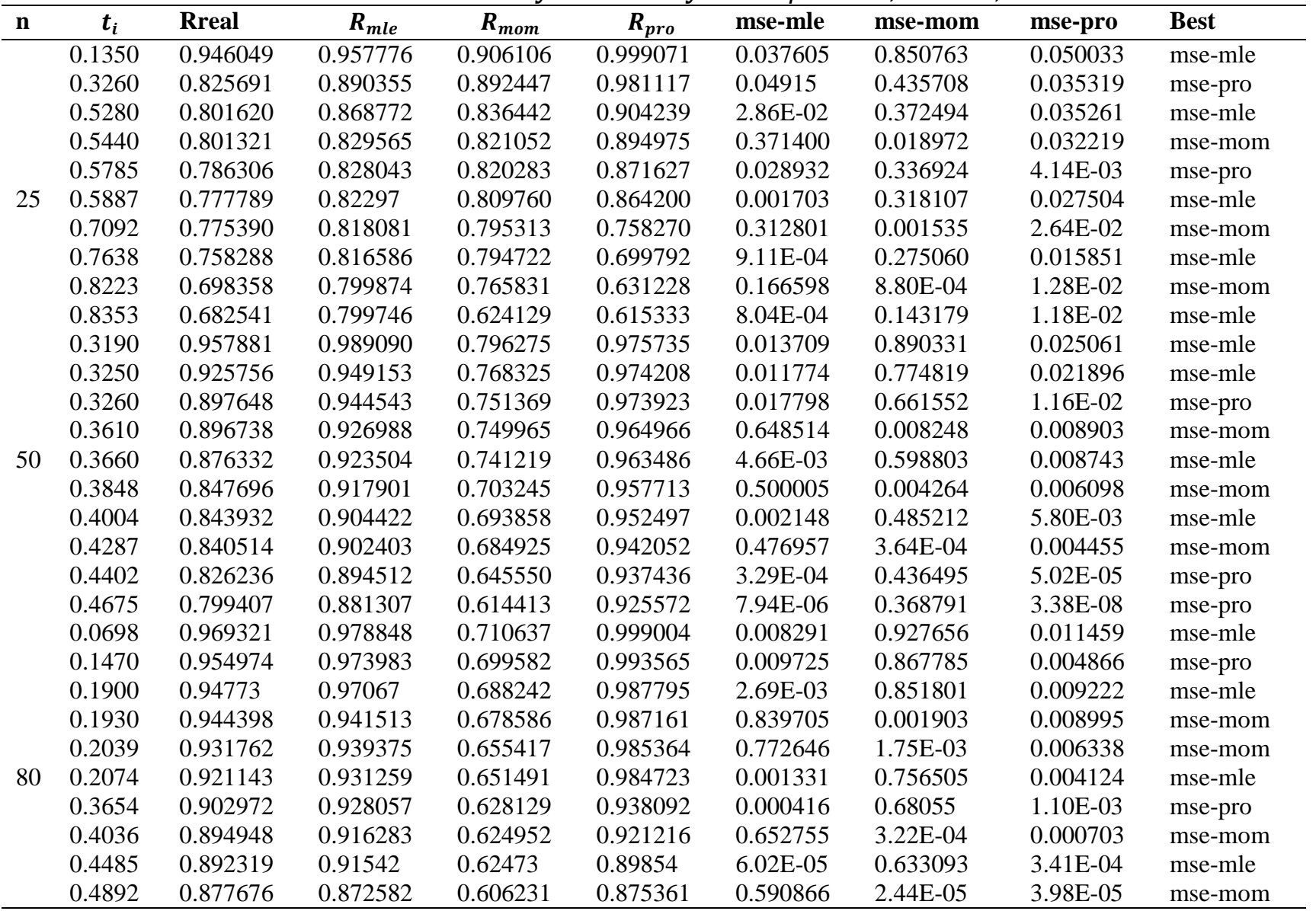

Table 8. Estimator Fuzzy Reliability when $\beta=2.5, \alpha=1.2, \tilde{k}=0.7$

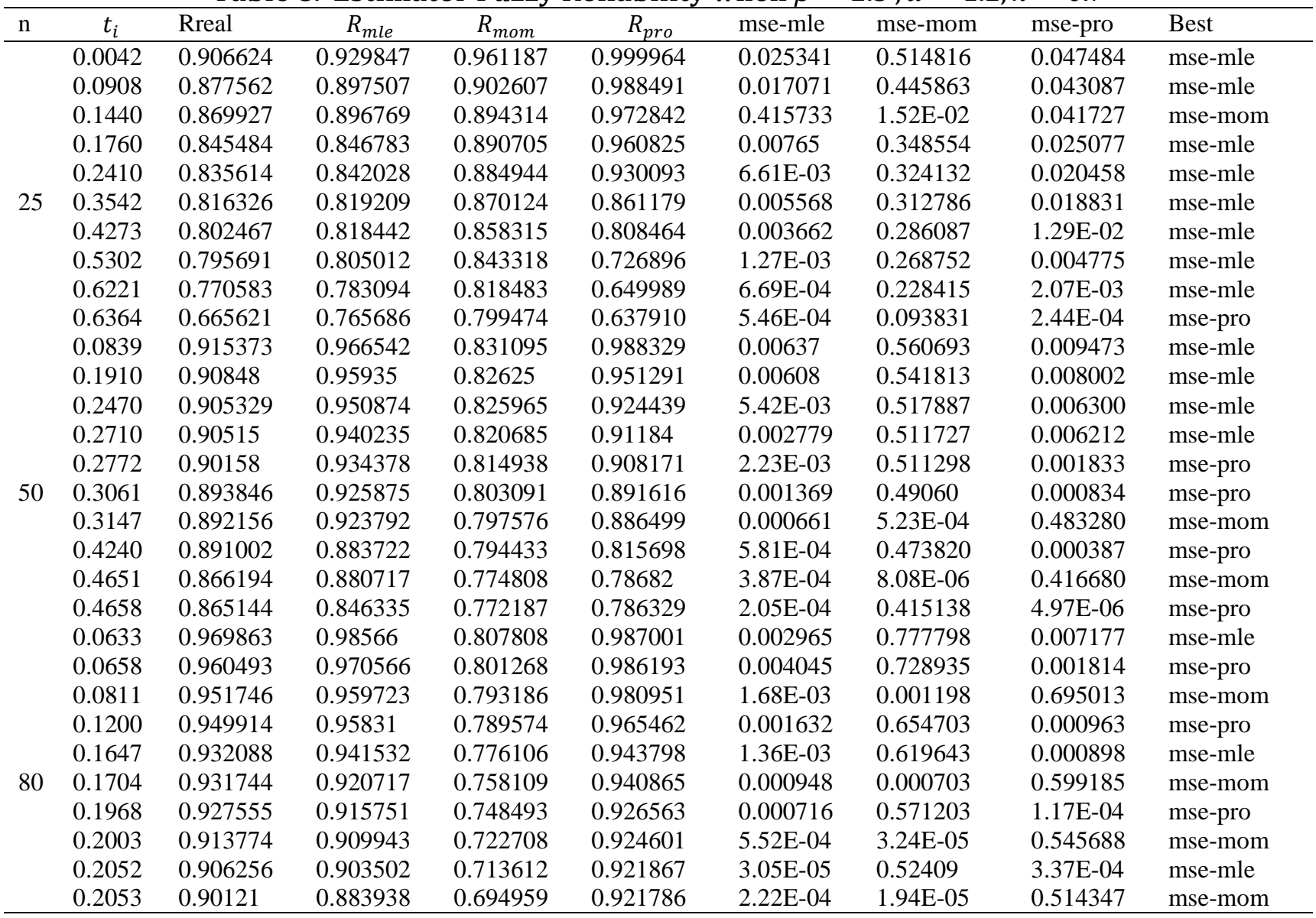




\section{Conclusion:}

From Table (1), the results of simulation .indicate that the best first fuzzy estimators of reliability function when $(\mathrm{n}=25)$ and $(\mathrm{n}=50)$ and $(\mathrm{n}=80)$, is Maximum Likelihood Estimator (MLE) with percentage $(63.33 \%)$, and second moment $\left(\widehat{\boldsymbol{R}}_{\text {mom }}\right.$ with percentage $\left.20.000 \%\right)$, and finally $\left(\widehat{\widehat{\boldsymbol{R}}}_{\text {proposed }}\right.$ is best with percentage $16.666 \%)$. From these results, it is concluded that $\widehat{\widehat{\boldsymbol{R}}}_{\boldsymbol{M L}}$ is first best and $\widehat{\boldsymbol{R}}_{\boldsymbol{m o m}}$ is the second best and finally $\widehat{\boldsymbol{R}}_{\text {proposed }}$ is the third best estimator.

Andfrom Table (2), the results indicate that the first best one estimator is the proposed since it has the best percentage(40\%), and second one is MLE with percentage $(36.666 \%)$, and third one is moment estimators since it has percentage $(23.333 \%)$.

Also from Table (3), the results of simulation indicate that, the first best fuzzy reliability estimator is (MLE) since it has the percentage $(50 \%)$ or $\left(\frac{15}{30}\right) \%$, and the second best estimator for fuzzy reliability is proposed with percentage $(26.666 \%)$ or $\left(\frac{8}{30}\right) \%$, while third one is moment estimator, has the percentage $(23.333 \%)$ or $\left(\frac{7}{30}\right) \%$.

The next tables summarize the results of simulation as:
Table 9. Summary of percentage of preference from each tables $(1,2, \ldots, 8)$ for three different estimators

\begin{tabular}{cccc}
\hline Tables & \% MLE & \% MOM & \% Proposed \\
\hline Table (1) & 63.333 & 20 & 16.66 \\
Table (2) & 36.666 & 23.34 & 40 \\
Table(3) & 50.000 & 23.333 & 26.666 \\
Table (4) & 53.333 & 23.33 & 23.33 \\
Table(5) & 40 & 26.66 & 33.33 \\
Table(6) & 26.666 & 50 & 23.333 \\
Table(7) & 43.333 & 33.333 & 23.333 \\
Table(8) & 50.000 & 23.333 & 26.333 \\
\hline
\end{tabular}

From the summary of results in Table (9), it is concluded that MLE is best with percentage(50\%), and then proposed is best with (25\%) and also MOM is best with $(25 \%)$.

\section{Conflicts of Interest: None.}

\section{References:}

1. Hussian MA. Fuzzy reliability estimation based on exponential ranked set samples. IJCMS.2017;1 (12):31-42.

2. Elbatal E M. Transmuted Quasi Lindly Distribution: A Generalization of the Quasi Lindly Distribution.IJSES.2013; 18(2):59-70.

3. Mohmoudi E. Zakerzadeh H. Generalized PoissonLindley Distribution. CSTM, 2010;39(10):17851798.

4. Zeghdoudi H, Nedjar S A. pseudo lindley distribution and it is Application, African Journal of Mathematics and Computer Science Research. AJMCSR.2016; 11(1):923-932.

5. Sankaran M. 275.note: The Discrete Poisson- Lindley Distribution, international Biometrics society. IBS, 1970; 26(1);145-149.

6. Shanker R, Mishra A, A Quasi Lindley Distribution. AJMCSR. 2013;6(4):64-71.

7. Zakerzadeh H, Dolati A. Generalization of lindley distribution.JME. 2009; 3(2) ;: 1-17.

8. Nedjar S, Zeghdoudi H. Gamma Lindley Distribution and its application. JAPS,2016;11(1):129-138.

9. Dutta P, Borah M. A study on Some properties of Poisson Size -Biased Quasi Lindly Distribution.IOSR-JM, 2015; 11 (3):23-28.

10. Shanker R, Shukla K . A Three parameter lindley distribution. AJMS,2017;7(1):15-26 
مناقشة عدة مقدرات للمعولية الضبابية لتوزيع احتمالي مختلط بواسطة المحاكاة

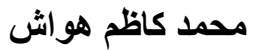

كلية دجلة الجامعة الأهلية، بغداد، العراق.

الخلاصة:

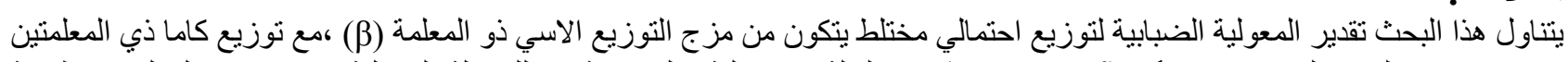

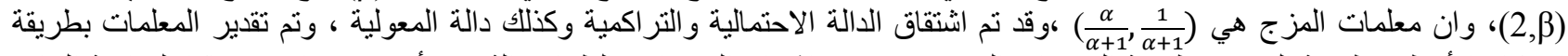

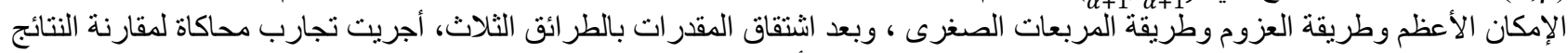

و إيجاد مقدر المعولية الضبابي الذي يحقق اصغر منوسط مربعات خطأ.

الكلمات المفتاحية:مقدرات الإمكان الأعظم ،متوسط مربعات الخطأ، نوزيع احتمالي مختلط ،مقدرات العزوم ،مقدرات مقترحة. 